\title{
PROPUESTA DE UN INDICADOR DE CAPACIDAD DE ABSORCIÓN DEL CONOCIMIENTO (ICAC-COL): EVIDENCIA EMPÍRICA PARA EL SECTOR SERVICIOS EN COLOMBIA*
}

\author{
CARLOS HERNÁN GONZÁLEZ CAMPO** \& ANDREA HURTADO AYALA*** \\ UNIVERSIDAD DEL VALLE
}

Recibido/ Received/ Recebido: 06/12/13 - Aceptado/ Accepted / Aprovado: 29/05/14

\begin{abstract}
Resumen
La "capacidad de absorción" es una capacidad dinámica reconocida por desarrollar habilidades de aprendizaje y explotación del conocimiento externo. Esta investigación propuso un Indicador de Capacidad de Absorción para Colombia denominado (ICAC-Col), utilizando los datos de la Encuesta de Desarrollo e Innovación Tecnológica realizada en Colombia, EDITS III para el sector servicios. Los resultados arrojan un nivel insuficiente de capacidad de absorción del conocimiento en el sector servicios, resaltando los sectores de transporte aéreo y servicios de gestión ambiental por poseer mayores capacidades para administrar el conocimiento, reflejando la necesidad de políticas públicas que impulsen actividades de gestión del conocimiento.

Palabras clave: Capacidad de absorción, Capacidades dinámicas, Gestión del conocimiento, Gestión de la innovación.
\end{abstract}

\section{PROPOSAL FOR AN INDICATOR OF KNOWLEDGE CAPACITY (ICAC-COL): EMPIRICAL EVIDENCE FOR SERVICES SECTOR IN COLOMBIA}

\begin{abstract}
"Absorption capacity" is a recognized dynamic capability because it develops learning skills and external knowledge exploitation. This research proposed an Absorption Capacity Indicator for Colombia called (ICAC-Col), using data from the Technological Innovation and development survey made in Colombia, EDITS III for services sector. The results show an insufficient level of knowledge absorption capacity in the services sector, however highlighting air transport sector and environmental management services because these have a greater ability to manage knowledge, reflecting the need for public policies to promote knowledge management activities.

Keywords: Absorption capacity, Dynamic capability, Knowledge management, Innovation management.

Artículo de investigación científica y tecnológica como resultado de la revisión teórica y empírica realizada durante el desarrollo de la tesis de grado para optar al título de Maestría en Ciencias de la Organización de la Universidad del Valle.

**: PhD en administración-Universidad EAFIT, Magister en Ciencias de la Organización-Universidad del Valle. Profesor e investigador Universidad del Valle. Director del Grupo de investigación Humanismo y Gestión de la Universidad del Valle. Correo electrónico: carlosh.gonzalez@correounivalle.edu.co. Tel: 5185760. Dirección postal: Calle 4B No 36-00, Universidad del Valle, Oficina 3012.

*** Maestra en Ciencias de la Organización (c), Economista y Contadora Pública de la Universidad del Valle. Asistente de docencia y miembro del Grupo de Investigación Humanismo y Gestión de la Universidad del Valle. Correo electrónico: andrea.hurtado.ayala@ correounivalle.edu.co. Tel: 5185760. Dirección postal: Calle 4B No 36-00, Cali, Valle.
\end{abstract}




\title{
PROPOSTA DE UM INDICADOR DE CAPACIDADE DE ABSORÇÃO DO CONHECIMENTO (ICAC-COL): EVIDÊNCIA EMPÍRICA PARA O SETOR SERVIÇOS NA COLÔMBIA
}

\begin{abstract}
Resumo
A "capacidade de absorção" é uma capacidade dinâmica reconhecida por desenvolver habilidades de aprendizagem e exploração do conhecimento externo. Esta pesquisa propôs um Indicador de Capacidade de Absorção para Colômbia denominado (ICAC-COL), utilizando os dados da Enquete de Desenvolvimento e Inovação Tecnológica realizada na Colômbia, EDITS III para o setor serviços. Os resultados apresentam um nível insuficiente de capacidade de absorção do conhecimento em setor serviços, ressaltando os setores de transporte aéreo e serviços de gestão ambiental por possuir maiores capacidades para administrar o conhecimento, refletindo a necessidade de políticas públicas que impulsionem atividades de gestão do conhecimento.

Palavras chave: Capacidade de absorção, Capacidades dinâmicas, Gestão do conhecimento, Gestão da inovação.

González, C. \& Hurtado, A. (2014) Propuesta de un Indicador de Capacidad de Absorción del Conocimiento (ICAC-Col): evidencia empírica para el sector servicios en Colombia. En: Revista de la Facultad de Ciencias Económicas de la Universidad Militar Nueva Granada. rev.fac.cienc.econ, XXII (2).
\end{abstract}

JEL: O31, O32, M10.

\section{Introducción}

El éxito en el desempeño empresarial se explica a partir de la creación de ventajas competitivas, buscando establecer una diferencia clara con respecto a sus competidores y que sea considerada importante por parte de los clientes. Cuando una empresa busca crear ventajas competitivas, debe enfocarse en reconocer las capacidades organizacionales que producen la generación de productividad y crecimiento (Jansen, Van Den Bisch \& Volberda, 2005; Kane, 2010). Específicamente, el aprendizaje y el conocimiento son algunas de las variables que tienen un rol estratégico en el desarrollo de nuevas capacidades (Sánchez, Heene \& Thomas, 1996).

El desempeño superior de las empresas exitosas sólo son explicadas a partir de las ventajas competitivas (Álvarez, 2003) y para Michael Porter (1985), la base del éxito del desempeño de las empresas radica en la ventaja competitiva, destacando que la innovación es el factor más importante y fuente de ventaja competitiva (Dávila, Epstein \& Shelton, 2005; Walsh, Lynch \& Harrington, 2011).

Así, los procesos relacionados a la generación de ventajas competitivas, no sólo permiten mejorar los márgenes de utilidad al facilitar su supervivencia y consolidación, sino que al mismo tiempo contribuyen al sostenimiento del modelo de negocio (Ubeda \& Moslares, 2008); por tanto, el aumento en la velocidad con que se dan los cambios tanto en la tecnología como en los mercados, consideran la innovación como aquella variable imprescindible en la creación y consolidación de ventajas competitivas que permitirán la subsistencia de las organizaciones (Díaz de Santos, 1997; Rodríguez, 1999).

Consecuentemente debe considerarse la realidad de las entidades productivas, las cuales no tienen más opción que innovar ya que los tiempos de exclusividad de la ventaja competitiva de sus productos o 
servicios en el mercado, cada vez son más cortos (Medina, 2013).

La Teoría de las Capacidades Dinámicas destaca la importancia de la armonización de los recursos y capacidades de le empresa con los cambios en el entorno, resalta la existencia de la capacidad de absorción del conocimiento considerada una capacidad dinámica que busca que las organizaciones se adapten al ambiente a través de procesos de transferencia de conocimiento que afectan su desempeño, y que son primordiales para conservar una ventaja competitiva sostenible (Zollo \& Winter, 2002).

En Colombia, los estudios relacionados con el tema de la gestión del conocimiento en las empresas se basan en el análisis de la formación del capital humano (Lugones, Gutti \& Le Clech, 2007). Estos esfuerzos parecen no ser suficientes para determinar el verdadero nivel de capacidad de absorción del conocimiento de las empresas colombianas, y aunque existen variables como, la tasa de alfabetización y el desarrollo de una base científica y tecnológica, que miden el nivel de conocimiento en los países, no representan la multidimensionalidad del término capacidad de absorción del conocimiento expuestas por los autores que plantearon este concepto (Cohen \& Levinthal, 1990; Lane \& Lubatkin, 1998; Van den Bosch, Volberda \& De Boer, 1999). Así mismo, los informes regionales de innovación, ciencia y tecnología, no se aproximan a medir la capacidad de absorción del conocimiento como una capacidad dinámica desarrollada en las organizaciones.

La principal aproximación de variables que pueden medir la gestión del conocimiento en las organizaciones en Colombia, es la inversión en Actividades Científicas, Tecnológicas y de Innovación (ACTI), considerada dentro de la Encuesta de Desarrollo e Innovación Tecnológica EDITS III realizada en el año 2011 para las empresas del Sector Servicios en Colombia y EDIT V realizada en el año 2010 para la industria manufacturera.

Hasta la fecha, en esta investigación no se evidenciaron estudios centrados específicamente en analizar la capacidad de absorción del conocimiento de las empresas en Colombia, tampoco existe el análisis de la relación con otros factores como la innovación, la competitividad y los resultados empresariales. Por esta razón, en los resultados de este artículo se plantea un indicador que mida la capacidad de absorción del conocimiento de las empresas colombianas, basado en el concepto de Zahra \& George (2002), para establecer con mayor claridad cómo se comportan las empresas colombianas respecto al desarrollo de dicha capacidad.

Este artículo es el resultado de una investigación, y está dividido en cuatro secciones: la primera corresponde al marco teórico y la evidencia empírica sobre el tema, en segundo lugar, se incluye la metodología utilizada en el estudio, posteriormente son presentados resultados, conclusiones y limitaciones de la investigación.

\section{Revisión de literatura}

Teniendo en cuenta la necesidad de las organizaciones por acercarse más a las demandas del mercado y del cliente en general, las empresas hacen grandes esfuerzos por invertir en procesos de gestión del conocimiento e innovación que les permita acceder a nuevos mercados o mantenerse en ellos. Esta obligación de conservar su posición en el mercado, promueve la búsqueda de recursos y de capacidades para sobrevivir y competir, propiciando la generación de conocimiento y su transferencia, así como el acceso a información valiosa. El fundamento teórico es el surgimiento de la Teoría de las Capacidades Dinámicas, sus principales características y exponentes, para centrarse en el análisis de la capacidad de absorción del conocimiento que según la evidencia empírica es una capacidad dinámica en las organizaciones.

\subsection{Teoría de las Capacidades Dinámicas}

El Enfoque Basado en Recursos surge a partir de los planteamientos de Penrose (1959) quien propone que el núcleo de la empresa debe basarse en los recursos que emplea y en las capacidades implementadas para el uso de dichos recursos. Penrose (1959) también hace énfasis en la importancia de la experiencia del empresario como recurso, al igual que del conocimiento que posee sobre el uso de los recursos existentes. 
A partir de los planteamientos de Penrose (1959), el Enfoque Basado en Recursos presentan nuevos avances. Rumelt (1984) encuentra en los estudios sobre la estructura industrial, la existencia de diferentes empresas con diversos usos de recursos y capacidades que conducen a resultados empresariales diferentes. Por su parte Wernerfelt (1984), Barney (1991) y Teece (1990) confirman que un sector económico se caracteriza por la heterogeneidad empresarial y la existencia de diversos niveles de desempeño asociados con diferentes tipos de estrategias empresariales.

A partir de este enfoque, la empresa se considera como una combinación de recursos tangibles $e$ intangibles, como activos, tecnologías, habilidades, conocimientos, etc., distinguiendo entre recursos de carácter tangible e intangible, para centrarse en los intangibles, basados en la información y el conocimiento, como soporte de las acciones competitivas de la empresa.

En este sentido, el trabajo de Wernerfelt (1984), plantea la importancia del conjunto de recursos de una empresa, enfocándose en los recursos internos como activos, información y conocimiento, y la forma como estos son adquiridos, controlados y desarrollados por parte de la empresa, permitiendo la creación de estrategias que mejoren su ventaja competitiva (Barney, 1991). Este enfoque cuestiona que el desarrollo de las ventajas competitivas deba depender de la dinámica del mercado, planteando que las ventajas competitivas surgen cuando existe heterogeneidad de recursos en las empresas y ésta alcanza sostenibilidad en el tiempo, permitiendo generar rentabilidad a largo plazo. Sin embargo, esta heterogeneidad en la dotación de recursos hace parte de un enfoque restrictivo y estático, si se considera que la transformación de recursos en resultados requiere de la existencia de capacidades organizacionales que son fundamentales para conseguir una ventaja competitiva, y que constituyen el aspecto dinámico del enfoque (Amit \& Schoemaker, 1993).

Así surge la Teoría de las Capacidades Dinámicas, con el propósito de dinamizar el enfoque basado en los recursos, partiendo de la necesidad de una continua renovación de los recursos y capacidades de una empresa de acuerdo a los cambios del mercado (Teece, Pisano \& Shuen, 1997; Eisenhardt \& Martin, 2000).

El concepto de capacidad dinámica surge como aquellas rutinas requeridas para que la empresa se adapte a los cambios del mercado (Teece \& Pisano, 1994), rutinas o actividades que deben enfocarse externamente (Ahuja \& Katila, 2001). Según Eisenhardt \& Martin (2000), las capacidades dinámicas varían de acuerdo al nivel de dinamismo del mercado, donde mercados altamente cambiantes deben enfocarse en crear nuevo conocimiento, de allí que las primeras definiciones se presenten en los años 90 , con continuos cambios en el contexto competitivo como producto de la rápida extensión de la globalización en los mercados.

Sin embargo, es a partir del trabajo de Teece et al. (1997) que la Teoría de las Capacidades Dinámicas toma fuerza, con la definición de capacidades dinámicas como "la habilidad de la empresa para integrar, construir y reconfigurar competencias internas y externas para adaptarse rápidamente a los cambios del mercado, reflejando la habilidad de la empresa para conseguir nuevas formas para alcanzar ventajas competitivas a partir de ciertas posiciones dadas por el mercado" (Teece et al., 1997, 516). Estos autores, señalan que los procesos organizacionales se clasifican según su función de integración, aprendizaje, reconfiguración y transformación de los recursos y competencias internas y externas con el fin de crear nuevas competencias (Amit \& Schoemaker, 1993). Teece (2007) complementa este concepto, afirmando que las capacidades dinámicas también permiten descubrir y aprovechar las oportunidades, y mantener la competitividad por medio del mejoramiento y reconfiguración de sus activos.

A partir de la evidencia empírica sobre la Teoría de las Capacidades Dinámicas, los autores buscan revelar los procesos que componen dichas capacidades, partiendo de las características comunes que presentan las capacidades dinámicas en las diferentes empresas. Wang \& Ahmed (2007) intentan hacer una recopilación de las diferentes definiciones, identificando que la gran mayoría de autores se refiere a recursos, procesos y capacidades (Teece et al., 1997; 
Eisenhardt \& Martin, 2000; Teece, 2007) sin llegar a un único consenso. Sin embargo, las capacidades organizacionales se caracterizan por convertir las ventajas internas generadas por los recursos tangibles y/o intangibles utilizados por la organización, en ventajas a nivel de mercado.

Según Prahalad \& Hamel (1990) las capacidades surgen también por el aprendizaje organizacional, en espacial el relacionado con la coordinación de las actividades de producción y la integración de nuevas tecnologías. Adicionalmente, la creación de capacidades no solo consiste en acoplar un conjunto de recursos, sino que implican una labor de coordinación entre las personas y los recursos que se desarrolla en las rutinas organizacionales, consideradas como el conjunto de relaciones que implican ciertos procesos de aprendizaje constituidos por una secuencia de acciones individuales coordinadas (Grant, 1996).

Adicionalmente, las capacidades también se basan en recursos intangibles como el conocimiento interno y externo de la empresa, y por esta razón se hace necesario desarrollar una capacidad de crear y transferir dicho conocimiento (Ventura, 1996). Wang \& Ahmed (2007) a partir de la evidencia empírica existente, encuentran tres tipos de capacidades dinámicas: la capacidad de adaptación, la capacidad de innovación y la capacidad de absorción.

La capacidad de adaptación consiste en la habilidad empresarial para identificar y aprovechar las oportunidades del mercado basándose en la estrategia empresarial (Miles \& Snow, 1978; Hooley, Lynch \& Jobber, 1992). La capacidad de innovación es la habilidad empresarial para desarrollar nuevos productos y/o procesos por medio de una estrategia orientada hacia la innovación (Hurley \& Hult, 1998; Petroni, 1998; Calantone, Cavusgil \& Zhao, 2002; Zhao et al., 2005). Finalmente, es importante resaltar que la capacidad de absorción es la habilidad empresarial para reconocer el valor proveniente de la información o conocimiento externo, para luego asimilarlo y aplicarlo con fines comerciales (Cohen \& Levinthal, 1990). Este artículo se centra en estudiar esta capacidad de absorción para las empresas del sector servicios en Colombia, partiendo de la evidencia empírica relacionada en el siguiente apartado.

\subsection{Evolución del concepto de capacidad de absorción del conocimiento}

La capacidad de absorción del conocimiento es un proceso determinante para la obtención de ventajas competitivas por parte de una organización, teniendo en cuenta que constituye una habilidad con tres funciones esenciales: adquirir conocimiento externo, integrarlo con el conocimiento existente, $y$ transformarlo en nuevo conocimiento, tres funciones que responden a su condición de capacidad dinámica, porque a partir de ellas se generan nuevas capacidades y rutinas organizacionales o se reconfiguran las ya existentes (Woiceshyn \& Daellenbach, 2005).

En el contexto organizacional, Cohen \& Levinthal (1990) definen el conocimiento como un conjunto de habilidades de aprendizaje, o acumulación de saberes que se van formando según las capacidades de las organizaciones. Entre las principales contribuciones de Cohen \& Levinthal (1989; 1990) sobresale la importancia de la innovación y el aprendizaje como parte de los procesos de $\mathrm{I}+\mathrm{D}$ de la empresa, teniendo en cuenta que hay un desarrollo en la capacidad de asimilar y explotar nuevo conocimiento externo. Estos primeros estudios sobre el tema, muestran que la capacidad de absorción depende en gran medida del nivel de conocimiento previo, identificando a las ciencias cognitivas y del comportamiento como el primer grupo de antecedentes que sustentan la asimilación de información en función de los conocimientos existentes.

Cohen \& Levinthal (1990) introducen el concepto capacidad de absorción definido como la habilidad que poseen las empresas para adquirir, asimilar y aplicar el conocimiento externo con fines comerciales, adicionalmente, estos autores indican que la capacidad de absorción de una empresa depende de tres aspectos: la relación entre el conocimiento interno y externo, las relaciones interorganizacionales, y las relaciones interpersonales en las áreas de la organización. En la Tabla 1 son presentadas algunas de las principales definiciones dadas para la capacidad de absorción del conocimiento. 
Tabla 1. Conceptos capacidad de absorción ${ }^{1}$

\begin{tabular}{|c|c|}
\hline Referente bibliográfico & Definición \\
\hline Cohen \& Levinthal (1990) & $\begin{array}{l}\text { "Habilidad para reconocer el valor de la nueva información externa, asimilarla, y aplicarla con fines comerciales". } \\
\text { (Cohen \& Levinthal, 1990, 128) }\end{array}$ \\
\hline Mowery \& Oxley (1995) & $\begin{array}{l}\text { "Habilidades que reflejan la necesidad de negociar los componentes tácitos de la tecnología trasferida y modificar } \\
\text { una fuente externa de tecnología para aplicaciones de tipo doméstico". (Mowery \& Oxley, 1995, 80) }\end{array}$ \\
\hline Lane \& Lubatkin (1998) & $\begin{array}{l}\text { "Habilidad relativa de una empresa para valorar, asimilar y aplicar el nuevo conocimiento de una empresa". (Lane } \\
\& \text { Lubatkin, 1998, 473) }\end{array}$ \\
\hline Dyer \& Singh (1998) & $\begin{array}{l}\text { "La capacidad de absorción de un socio específico, se refiere a la idea de que una empresa haya desarrollado la } \\
\text { habilidad de reconocer y asimilar conocimientos valiosos de un socio de una alianza particular. Esta capacidad } \\
\text { implicaría la implementación de un conjunto de procesos inter-organizacionales que permiten a las empresas que } \\
\text { colaboran, identificar sistemáticamente el valioso know-how y luego transferirlo a través de fronteras organizativas". } \\
\text { (Dyer \& Singh, 1998, 665). }\end{array}$ \\
\hline Zahra \& George (2002) & $\begin{array}{l}\text { "Conjunto de rutinas organizacionales y procesos estratégicos por los que las empresas adquieren, asimilan, trans- } \\
\text { forman y explotan conocimiento con la intención de crear valor". (Zahra \& George, 2002, 186) }\end{array}$ \\
\hline $\begin{array}{l}\text { Minbaeva, Pedersen, Björkman, Fey \& } \\
\text { Park (2003) }\end{array}$ & $\begin{array}{l}\text { "La capacidad de absorción es una función tanto de las competencias y la motivación. En otras palabras, la capa- } \\
\text { cidad de absorción se examina a lo largo de las siguientes dimensiones: la capacidad de reconocer el valor del } \\
\text { conocimiento nuevo, asimilar y comercializarlo y conducir a ello". (Minbaeva et al., 2003, 2) }\end{array}$ \\
\hline Lane, Koka \& Pathak (2006) & $\begin{array}{l}\text { "Habilidad de una empresa para utilizar el conocimiento externo por medio de tres procesos: (1) el reconocimiento } \\
\text { del tipo de nuevo conocimiento ofrecido por la empresa emisora, (2) la asimilación del conocimiento nuevo y } \\
\text { valioso, (3) la utilización del conocimiento asimilado para crear conocimiento nuevo con fines comerciales". (Lane, } \\
\text { Koka \& Pathak, 2006, 776) }\end{array}$ \\
\hline Todorova \& Durisin (2007) & $\begin{array}{l}\text { "La habilidad para aprender, para absorber conocimiento externo, que depende en gran medida de la habilidad para } \\
\text { identificar y evaluar el nuevo conocimiento externo". (Todorova \& Durisin, 2007, 776) }\end{array}$ \\
\hline Liao, Wu, Hu \& Tsuei (2010) & $\begin{array}{l}\text { "La capacidad de absorción es la capacidad de evaluar y utilizar el conocimiento fuera de la organización con el fin } \\
\text { de identificar el ambiente organizacional". (Liao et al., 2010,760) }\end{array}$ \\
\hline Poldahl (2012) & $\begin{array}{l}\text { "Es la capacidad de la empresa para explotar el conocimiento exterior de un orden más intermedio, como los resul- } \\
\text { tados de la investigación básica que proporcionan la base para la investigación y el desarrollo aplicado posterior". } \\
\text { (Poldahl, } 2012,2 \text {.) }\end{array}$ \\
\hline
\end{tabular}

Posterior al trabajo de Cohen \& Levinthal (1990), Mowery \& Oxley (1995) definen que la capacidad de absorción está basada principalmente en las inversiones en la formación científica y técnica, y en las políticas económicas que refuerzan la competencia entre las empresas nacionales a través de los canales para la transferencia de tecnología hacia la organización. Lane \& Lubatkin (1998), realizan una reconceptualización al referirse a la "capacidad de absorción relativa", como la habilidad de una empresa para aprender de otras empresas, dependiendo de similitudes entre empresas en aspectos como: la base de conocimiento, la estructura organizacional, la política de compensación y la lógica dominante. En el mismo año, Dyer \& Singh (1998) se refieren a la capacidad de absorción de un socio-específico como el desarrollo de la capacidad de reconocer y asimilar conocimientos valiosos de una alianza especial. Esta capacidad implica la implementación de un conjunto de procesos interinstitucionales que permiten a las empresas colaboradoras identificar sistemáticamente conocimiento valioso, para luego transferirlo a través de fronteras organizacionales.

En este sentido, Zahra \& George (2002) redefinen la capacidad de absorción como una capacidad dinámica relacionada con la creación y utilización de conocimiento que mejora la habilidad de una empresa para obtener y mantener una ventaja competitiva. Zahra \& George (2002) sugieren que la capacidad de absorción está dividida en dos partes: la capacidad potencial donde se adquiere conocimiento y

1 Fuente: Elaboración propia. 
se asimilan capacidades, y la capacidad realizada centrada en la transformación y explotación del conocimiento.

Minbaeva et al. (2003) proponen que la capacidad de absorción no debe ser analizada en dimensiones separadas, sino que debe tomarse como un efecto interactivo entre capacidad y motivación que permita la transferencia de conocimientos, y conduzca a una mayor utilidad de los conocimientos adquiridos.

Lane, Koka \& Pathak (2006) realizaron una revisión crítica de los conceptos que existían hasta el momento, encontrando que la mayoría de estudios han intentado ampliar la definición original de Cohen \& Levinthal (1990), pasando de enfocarse en la capacidad de absorción como una actividad de $\mathrm{I}+\mathrm{D}$, y centrándose en definirla como una capacidad dinámica.

Todorova \& Durisin (2007) basados en el concepto original de Cohen \& Levinthal (1990) y las dimensiones propuestas por Zahra \& George (2002), incorporan un nuevo componente, donde la transformación del conocimiento no es el paso posterior a la etapa de asimilación, sino que constituye un proceso alternativo que se vincula a la asimilación a través de diferentes caminos, incorporando la presencia de factores de contingencia de integración social como aspectos que deben influir tanto en la transformación como en los demás componentes: adquisición, asimilación, explotación.

Para Liao, Wu, Hu \& Tsuei (2010), la capacidad de absorción hace parte de un conjunto de procesos claves para la gestión del conocimiento, entre ellos, la captura, transferencia, uso, adquisición, colaboración, integración y experimentación, donde la adquisición del conocimiento es considerada como la primera actividad de aceptación del conocimiento desde el ambiente externo. Entre las definiciones más recientes, Poldahl (2012) plantea la frase "las dos caras de la inversión en $I+D$ ", donde las actividades de I+D no solo estimulan la innovación, sino también, la capacidad de las empresas para asimilar el conocimiento externo. Por ende, esta segunda cara es llamada capacidad de absorción, vista como un subproducto de la inversión en I+D que también puede desarrollarse como un subproducto de las actividades propias de la empresa.

Estas definiciones hacen parte de un extenso grupo de investigaciones enfocadas en redefinir y unificar el concepto de capacidad de absorción del conocimiento. Para esta investigación, tomando como base la consideración de la capacidad de absorción como una capacidad dinámica, se parte de la definición dada por Zahra \& George (2002) que capta los aspectos dinámicos que promueven el cambio organizacional.

\subsection{La capacidad de absorción como una capacidad dinámica}

Zahra \& George (2002) consideran que la capacidad de absorción está conformada por un conjunto de rutinas y procesos desarrollados por las empresas para adquirir, asimilar, transformar y explotar el conocimiento para producir una capacidad dinámica organizacional. Estas cuatro capacidades representan cuatro dimensiones de la capacidad de absorción que son diferentes pero complementarias. Estos autores sustentan su propuesta a partir de la distinción entre capacidades y capacidades dinámicas, definiendo las capacidades como un tipo de rutinas que gestionan los flujos de "inputs" originando diferentes opciones para producir resultados "outputs" (Zahra et al., 2000). Como complemento, las capacidades dinámicas están dirigidas a generar un cambio organizacional, porque son esencialmente estratégicas y definen el camino que deben seguir las empresas para evolucionar (Teece et al., 1997).

La definición de Zahra \& George (2002), sugiere que las cuatro capacidades organizacionales de adquisición, asimilación, trasformación y explotación, construyen conjuntamente la capacidad de absorción como una capacidad dinámica que influye en las habilidades de la empresa para crear y desarrollar los conocimientos necesarios para implantar nuevas capacidades que conducen a obtener ventajas competitivas.

Zahra \& George (2002) también plantean que la capacidad de absorción debe dividirse en potencial y realizada, donde la capacidad de absorción poten- 
cial está conformada por la capacidad de adquisición y asimilación del conocimiento externo, y la capacidad de absorción realizada se desarrolla en función de la transformación y explotación del dicho conocimiento. Ambos tipos de capacidad poseen roles complementarios, dado que no es posible que una se desarrolle sin la otra. Adicionalmente, al referirse a la forma de medir dichas capacidades, Zahra \& George (2002) aclaran que ambas son estimadas como un factor de eficiencia que sugiere que las empresas difieren en su capacidad para generar valor a partir de su base de conocimiento, debido a que sus capacidades para transformar y explotar el conocimiento también sufren variaciones.

Por último, desde Zahra \& George (2002) es posible concluir que la distinción entre capacidad potencial y realizada evidencia la ineficiencia de algunas empresas en el aprovechamiento de su capacidad potencial que impide mejorar sus resultados, demostrando que estos dos componentes en su conjunto contribu- yen a construir ventajas competitivas, identificando los roles necesarios para gestionar y desarrollar dichas capacidades.

Para efectos de esta investigación se toma el concepto de Capacidad de Absorción dado por Zahra \& George (2002) para asumir que dicha capacidad se construye a partir de las cuatro dimensiones mencionadas, más no se realiza una distinción entre capacidad potencial y realizada. En cuanto a la construcción del indicador que se desarrolla más adelante, cabe aclarar que al aporte central de Zahra \& Goerge (2002), es complementado por los estudios de Todorova \& Durisin (2007) y Flor \& Oltra (2008), con el propósito de definir cada una de las dimensiones y establecer las variables que componen el indicador acordes con la definición de cada dimensión. En la Tabla 2 se presentan las definiciones de cada dimensión de la capacidad de absorción que serán utilizadas en esta investigación.

Tabla 2. Dimensiones de la capacidad de absorción del conocimiento²

\begin{tabular}{|l|l|}
\hline ADQUISICIÓN & $\begin{array}{l}\text { Capacidad que posee la empresa para identificar y adquirir conocimiento externo } \\
\text { considerado útil e importante para las actividades de la empresa. De esta capacidad } \\
\text { hacen parte todas las rutinas que posean los atributos de intensidad y velocidad que } \\
\text { son los esfuerzos de la empresa por identificar y recopilar el conocimiento, y el atri- } \\
\text { buto de dirección que influye en la acumulación de conocimiento y la capacidad de } \\
\text { la empresa de continuar adquiriendo conocimiento externo (Zahra \& George, 2002) }\end{array}$ \\
\hline ASIMILACIÓN & $\begin{array}{l}\text { Capacidad de la empresa para interpretar y comprender el conocimiento externo } \\
\text { con las estructuras cognitivas existentes (Todorova \& Durisin, 2007). Esto implica } \\
\text { como lo menciona Flor \& Oltra (2008), el desarrollo de una habilidad para analizar } \\
\text { y obtener la información de los actores del como una forma de atraer y relacionar el } \\
\text { conocimiento externo con el existente en la empresa. }\end{array}$ \\
\hline TRANSFORMACIÓN & $\begin{array}{l}\text { Todorova \& Durisin (2007) describen la transformación como un proceso alternativo } \\
\text { a la asimilación que se produce cuando los nuevos conocimientos no pueden ser } \\
\text { alterados para ajustarse a los conocimientos existentes. Por lo tanto, la transforma- } \\
\text { ción es la capacidad para vincular el conocimiento nuevo a través de la construcción } \\
\text { de nuevos esquemas cognitivos. }\end{array}$ \\
\hline EXPLOTACIÓN & $\begin{array}{l}\text { Capacidad de las empresas basada en rutinas que permiten perfeccionar, ampliar } \\
\text { y aprovechar las competencias existentes o crear nuevas competencias (Zahra \& } \\
\text { George, 2002). }\end{array}$ \\
\hline
\end{tabular}

2 Fuente: Elaboración propia. 
A partir de estas descripciones, se platean las variables que medirán cada dimensión, seleccionado las variables equivalentes de acuerdo de acuerdo a lo definido para cada dimensión y basado en las medidas propuestas por Zahra \& George (2002), Todorova \& Durisin (2007) y Flor \& Oltra (2008).

\section{Metodología}

El propósito de esta investigación es plantear un indicador para medir el nivel de capacidad de absorción del conocimiento y aplicarlo en la estimación para el sector servicios en Colombia. Para ello, primero se describe la muestra de empresas a la que será aplicado el indicador, posteriormente, se describen la variables que conforman las medidas del indicador, y por último el planteamiento de la operación matemática que da origen al indicador de capacidad de absorción del conocimiento.

\subsection{Descripción de la muestra}

En esta investigación se utiliza la base de datos de la Encuesta de Desarrollo e Innovación Tecnológica (EDIT) realizada por el DANE, utilizando la versión EDIT-V realizada a las empresas del sector industrial manufacturero en Colombia para el periodo 20092010, y la versión EDITS-III aplicada a las empresas del sector servicios en Colombia durante el periodo 2010-2011. Este proyecto es liderado por el DANE con el apoyo del Departamento Nacional de Planeación (DNP) y el Departamento Administrativo de Ciencia, Tecnología e Innovación (COLCIENCIAS).

Esta encuesta está diseñada bajo un marco teórico y metodológico basado en los principales acuerdos sobre diseño, aplicación e interpretación de encuestas nacionales de innovación, logrados por la comunidad nacional e internacional. Su diseño, se encuentra inspirado en la metodología del Manual de Frascati y del Manual de Oslo, especificando las actividades empresariales que conducen a la creación, adaptación y difusión del conocimiento, así como a la innovación (DANE, 2011).

Para el caso de las empresas del sector servicios, se aplicó a 5.423 empresas que realizaron actividades de servicios en el año 2011, de las cuales se obtu- vo información de 5.038 empresas, que conforman 16 subsectores del sector de servicios (de acuerdo al CIIU) relevantes a la investigación (DANE, 2012). La recolección de la información para la encuesta EDITS-III se realiza mediante un muestreo no probabilístico con una población objetivo constituida por 16 subsectores que componen el sector servicios, seleccionados por su relevancia en la investigación, y por cumplir con los parámetros de inclusión determinados según el nivel de personal ocupado y los ingresos anuales de las empresas (DANE, 2013).

\subsection{Variables del indicador}

La capacidad de absorción del conocimiento se ha caracterizado por ser un concepto multidimensional, por estar medido a través del análisis de sus fases y dimensiones que la componen, conforme a las diferentes definiciones propuestas en la literatura (Lane \& Lubatkin, 1998; Todorova \& Durisin, 2007).

Sin embargo, el uso de indicadores ha sido uno de los métodos más comunes para medir cuantitativamente esta capacidad (Murovec \& Prodan, 2009; Flatten et al., 2011), pero estos indicadores consideran la capacidad de absorción como un constructo unidimensional, utilizando para su medida variables proxy como: la proporción del gasto en $\mathrm{I}+\mathrm{D}$ dividido como porcentaje de las ventas anuales (Cohen \& Levinthal, 1990; Tsai, 2001); la intensidad en el uso de I+D (De Jong \& Freel, 2010); la inversión en formación del personal en áreas técnicas y científicas (Mowery \& Oxley, 1995); entre otros. Considerando las definiciones de la capacidad de absorción como una habilidad organizacional conformada por un conjunto de dimensiones, este tipo de medidas unidimensionales no recogen el efecto de cada una de las fases o dimensiones de la capacidad de absorción.

\subsubsection{Fase adquisición}

De acuerdo a la escala construida por Zahra \& George (2002), esta fase es medida por tres ítems: i) realización de $\mathrm{I}+\mathrm{D}$ próximo al avance tecnológico, ii) realización de inversiones para el desarrollo de nuevos productos y tecnologías, iii) mantener un departamento de $\mathrm{I}+\mathrm{D}$ altamente calificado. A partir 
de estos ítems, se buscan variables que logren medir estas tres condiciones, clasificando dentro de esta fase las siguientes variables:

$\begin{aligned} \mathrm{X}_{1}= & \text { Inversión en } \mathrm{I}+\mathrm{D} \text { interna y externa: medida en } \\ & \text { miles de pesos. }\end{aligned}$

$\mathrm{X}_{2}=$ Inversión en transferencia tecnológica, ingeniería de diseño, y mercadeo de innovaciones: medida en miles de pesos.

\subsubsection{Fase asimilación}

En esta investigación se parte de la escala de Flor \& Oltra (2008) quienes toman como base el concepto de asimilación de Todorova \& Durisin (2007) que pretende medir la capacidad de análisis y compresión del nuevo conocimiento. Para ellos, Flor \& Oltra (2008) hace referencia a las habilidades para analizar y obtener información relevante de actores del mercado como competidores, clientes, proveedores y demás instituciones, dado que esta constituye la mejor forma para relacionar el nuevo conocimiento con el conocimiento existente. Esta habilidad se recoge en dos ítems: i) obtener información de los proveedores $e$ instituciones locales sobre nuevas técnicas y materia prima, y ii) obtener información de los clientes. En esta investigación, se toman las siguientes variables:

$\mathrm{X}_{3}=$ Cooperación con proveedores para la realización de actividades de Científicas, Tecnológicas y de Innovación (ACTI): medida por el número de empresas que cooperaron con proveedores.

$\mathrm{X}_{4}=$ Cooperación con instituciones para la realización de actividades de Científicas, Tecnológicas y de Innovación (ACTI): medida por el número de empresas que cooperaron con instituciones locales (entidades gubernamentales, universidad, centros tecnológicos, etc.)

$\mathrm{X}_{5}=$ Cooperación con clientes para la realización de actividades de Científicas, Tecnológicas y de Innovación (ACTI): medida por el número de empresas que cooperaron con clientes.

\subsubsection{Fase transformación}

En esta investigación se adapta la escala de Todorova \& Durisin (2007) que mide la capacidad de construir nuevas capacidades a partir del conocimiento adquirido, asumiendo que existe conocimiento nuevo que es incompatible con el conocimiento existente, y por lo tanto se requiere generar nuevas estructuras cognitivas, que para Flor \& Oltra (2008) son interpretadas como fuentes de innovación o de generación de nuevas ideas porque conducen a la construcción de las nuevas estructuras cognitivas. Para esto se utilizan los siguientes tres ítems: i) realización de cursos de formación, ii) uso de servicios de consultoría, iii) publicaciones científicas y técnicas. Como variables que hacen parte de estos ítems se toman las siguientes:

$\mathrm{X}_{7}=$ Formación y Capacitación: número de empleados que recibieron formación y/o capacitación por parte de las empresas.

$\mathrm{X}_{8}=$ Inversión en asistencia técnica y consultoría: medida en miles de pesos

\subsubsection{Fase explotación}

En esta dimensión se utilizan medidas asociadas con la capacidad de generar nuevas competencias a partir del conocimiento adquirido, asimilado y transformado, representado en las rutinas para crear nuevos productos o mejorar los existentes (Zahra \& George, 2002), prácticas de gestión que mejoren la aplicación del conocimiento (Todorova \& Durisin, 2007), entre otros. Según Zahra \& George (2002) sugieren medidas como el número de patentes, anuncios de nuevos productos, o duración del ciclo de desarrollo de productos. Por su parte, Flor \& Oltra (2008) consideran que los siguientes ítems tienen en cuenta las consideraciones realizadas por Zahra \& George (2002) para esta dimensión y que en últimas buscan establecer los resultados finales del proceso de adquisición, asimilación y transformación del conocimiento:

$\mathrm{X}_{6}=$ Registros de propiedad intelectual: medida por el número de registros de propiedad intelectual obtenidos por las empresas. 
$\mathrm{X}_{9}=$ Mejora en la calidad del producto o servicio: medida por el número de innovaciones importantes de este tipo llevadas a cabo por las empresas.

$\mathrm{X}_{10}=$ Ampliación gama de productos o servicios ofrecidos: medida por el número de innovaciones importantes de este tipo llevadas a cabo por las empresas.

\subsection{Validez de los constructos}

Cada una de estas cuatro fases o dimensiones constituyen cuatro constructos que son validados mediante la estimación de un análisis factorial exploratorio que permite determinar si los indicadores seleccionados se agrupan en un mismo factor o constructo (Nunally, 1978), permitiendo comprobar la medición del concepto de capacidad de absorción definido. Para la construcción de instrumentos de medición, el análisis factorial por componentes principales suele ser el más recomendado porque analiza toda la varianza de las variables (Nunally, 1978; Spector, 1992). Por otra parte en cuanto al tipo de rotación que permite realizar la transformación lineal para interpretar la varianza explicada de os factores (Nunally \& Bernstein, 1994), y la rotación utilizada en esta investigación es la varimax porque busca simplificar el número de factores (Nunally, 1978; Rennie, 1997), facilitando la interpretación del instrumento de medida y obteniendo constructos diferenciados.
Inicialmente se analizan los resultados del coeficiente KMO y de la prueba de esfericidad de Bartlett, el primero contrasta si las correlaciones parciales entre las variables son muy pequeñas, y para valores inferiores a 0,5 se invalida la utilización de análisis factorial con los datos muestrales que están siendo empleados (Hair et al., 1999). La prueba de esfericidad de Bartlett, contrasta la hipótesis nula de que la matriz de correlaciones es una matriz identidad, caso en el que no existirían correlaciones significativas entre variables, y el análisis factorial no sería adecuado (Hair et al., 1999), la hipótesis nula se rechaza para un nivel de significancia menor de 0,05 en el estadístico Chi-cuadrado.

De acuerdo a la Tabla 3, se obtiene un coeficiente KMO de 0,559 y un nivel de significancia del Chicuadrado inferior a 0,05 confirmando la pertinencia del análisis factorial.

Tabla 3. KMO y prueba de esfericidad de Bartlett $^{3}$

\begin{tabular}{|c|c|c|}
\hline & & Servicios \\
\hline \multicolumn{2}{|c|}{$\begin{array}{l}\text { Medida de Adecuación muestral } \\
\text { de Kaiser-Meyer-Olkin }\end{array}$} & 0,559 \\
\hline $\begin{array}{c}\text { Prueba de esfericidad } \\
\text { de Bartlett }\end{array}$ & $\begin{array}{c}\text { Chi-cuadrado } \\
\text { aproximado } \\
\text { gl } \\
\text { Sig. }\end{array}$ & $\begin{array}{c}119,661 \\
45 \\
0,000\end{array}$ \\
\hline
\end{tabular}

Tabla 4. Análisis factorial exploratorio ${ }^{4}$

\begin{tabular}{|c|c|c|c|c|c|c|c|c|}
\hline \multirow{5}{*}{ Factores } & \multicolumn{2}{|c|}{$\begin{array}{c}\text { F1 } \\
\text { Explotación }\end{array}$} & \multicolumn{2}{|c|}{$\begin{array}{c}\text { F2 } \\
\text { Asimilación }\end{array}$} & \multicolumn{2}{|c|}{$\begin{array}{c}\text { F3 } \\
\text { Transformación }\end{array}$} & \multicolumn{2}{|c|}{$\begin{array}{c}\text { F4 } \\
\text { Adquisición }\end{array}$} \\
\hline & Variable & Peso & Variable & Peso & Variable & Peso & Variable & Peso \\
\hline & $x 6$ & 0,542 & X3 & 0,923 & $\mathrm{X} 7$ & 0,849 & $x_{1}$ & 0,770 \\
\hline & X9 & 0,644 & $X 4$ & 0,878 & $\mathrm{X} 8$ & 0,961 & $\mathrm{X} 2$ & 0,798 \\
\hline & $\mathrm{X} 10$ & 0,888 & $\times 5$ & 0,954 & & & & \\
\hline \% varianza explicada & \multicolumn{2}{|c|}{34,280} & \multicolumn{2}{|c|}{27,125} & \multicolumn{2}{|c|}{18,082} & \multicolumn{2}{|c|}{12,743} \\
\hline \% varianza acumulada & \multicolumn{2}{|c|}{34,280} & \multicolumn{2}{|c|}{61,404} & \multicolumn{2}{|c|}{79,486} & \multicolumn{2}{|c|}{92,229} \\
\hline
\end{tabular}

\footnotetext{
Fuente: Elaboración propia.

4 Fuente: Elaboración propia.
} 
La Tabla 4 evidencia los resultados del análisis factorial exploratorio que confirma la existencia de las cuatro dimensiones de la capacidad de absorción. El primer factor resultante corresponde a la explotación, donde las tres variables de medición presentan una carga superior a 0,5 , garantizando que cada una de ellas pertenece al factor en el que ha quedado clasificado. Lo mismo sucede con el factor 2 que agrupa las variables que miden la asimilación, y el factor 4 que agrupa las variables que miden la adquisición. El factor 3 recoge las variables de medición de la transformación. Estos datos son tomados de la matriz de componentes rotados, donde se obtiene que las variables correspondientes a cada factor no presentan valores superiores a 0,5 en ninguno de los otros factores, permitiendo determinar que efectivamente cada variable pertenece a la dimensión descrita.

A partir de estos resultados, es verifica la fiabilidad de las diferentes dimensiones del constructo y sus indicadores. Cada una de estas cuatro fases o dimensiones constituyen cuatro constructos, validados el análisis de fiabilidad mediante el Alfa de Cronbach, coeficiente que según Nunally (1978), presenta valores satisfactorios cuando su valor es superior a 0,6 , indicando que la escala utilizada posee consistencia interna reflejada en la correlación entre los distintos ítems que componen el constructo, y demostrando que los componentes del constructo son equivalentes entre sí. La validación del constructo adquisición compuesto por dos ítems, es realizada mediante el coeficiente Alfa de Cronbach cuyo resultado es 0,647 . La validación del constructo asimilación se realiza mediante el coeficiente Alfa de Cronbach que genera un resultado de 0,738 , considerando que se encuentra en un nivel satisfactorio, Para el constructo transformación, el coeficiente Alfa de Cronbach arroja un valor de 0,652. El constructo explotación genera un coeficiente Alfa de Cronbach con un valor altamente satisfactorio de 0,878 .

\subsection{Cálculo del indicador}

Después de validar los constructos que conforman las dimensiones de la capacidad de absorción y sus indicadores, se plantea la fórmula para estimar el indicador de capacidad de absorción, partiendo de la metodología utilizada por Sullivan (1994), quienes destacan las bondades de la estimación de un indicador mediante una combinación lineal de las medidas individuales, alineados con los planteamientos de Nunnally (1978). Sullivan (1994) relaciona cada elemento del indicador con la puntuación de cada variable, y el indicador se define como la suma de las puntuaciones de todos los elementos que lo componen.

La estandarización del indicador, parte del Método de Re-escalamiento descrito por Schuschny \& Soto (2009), transformando los niveles de las variables para llevarlos al intervalo [0,1], estimando la distancia entre los valores máximos y mínimos de la variable en su conjunto. Este método permite unificar las diferentes unidades de medida, y evitar errores de sesgo de información de los datos. De esta forma, en cada dimensión se estima una razón que origine como resultado un valor entre 0 y 1 que indica el porcentaje de presencia de dicha dimensión de capacidad de absorción en las empresas, para luego estimar el indicador global como una combinación lineal, sumando el valor obtenido por cada dimensión, y estableciendo un rango de valores para medir el nivel de capacidad de absorción.

Teniendo en cuenta esta metodología se plantea el siguiente indicador:

$$
\begin{gathered}
I C A C-\mathrm{Col}=A D Q+A S I M+ \\
\text { TRANSF }+ \text { EXPLOT }
\end{gathered}
$$

Donde:

$A D Q=$ mide el grado de adquisición del conocimiento por medio de la sumatoria de la inversión en $\mathrm{I}+\mathrm{D}$ y en transferencia tecnológica como porcentaje de la inversión total en ACTI.

ASIM = mide el grado de asimilación del conocimiento por medio de la sumatoria de cada uno de los tipos de cooperación como porcentaje del número total de empresas que han cooperado con otros socios.

TRANSF $=$ mide el grado de transformación del conocimiento por medio de la sumatoria entre: la variable formación y capacitación como porcentaje del 
número de empleados de las empresas, y la variable inversión en servicios técnicos y consultoría como porcentaje del total de inversión en ACTI.

EXPLOT $=$ mide el grado de explotación del conocimiento por medio de la sumatoria entre: la variable registros de propiedad intelectual como porcentaje del número de empresas, y las variables de mejora y ampliación del producto y/o servicio como porcentaje del número total de innovaciones realizadas por las empresas.

Adicionalmente, para fijar la escala de análisis del nivel de capacidad de absorción, partiendo de Schuschny \& Soto (2009), la escala es transformada como un indicador porcentual que permita comparar los resultados entre subsectores, donde $0 \%$ implica una capacidad de absorción nula y $100 \%$ es el máximo nivel de capacidad de absorción. Para tal fin, es medida la distancia entre los valores máximos y mínimos de cada variable, evitando problemas de sesgos en las estimaciones (Schuschny \& Soto, 2009).

\section{Resultados}

Los resultados obtenidos para el sector servicios en Colombia son resumidos en la Tabla No. 5, que muestra un indicador de capacidad de absorción del conocimiento de 1,65 , que implica este sector solo tienen una capacidad organizacional máxima del $41 \%$ para absorber el conocimiento externo.

Tabla 5. Capacidad de absorción del conocimiento del sector servicios en Colombia ${ }^{5}$

\begin{tabular}{|c|c|c|c|c|c|c|c|c|c|c|c|}
\hline CIIU & Nombre del subsector & Adquisición & & Asimilación & \multicolumn{3}{|c|}{ Transformación } & Explotación & \multicolumn{2}{|c|}{ Indicador } & \multirow{2}{*}{$\begin{array}{c}\% \\
38 \%\end{array}$} \\
\hline 40 & $\begin{array}{l}\text { Suministro de electricidad, gas, vapor y } \\
\text { agua caliente }\end{array}$ & 0,07 & $7 \%$ & 0,38 & $38 \%$ & 0,3 & $30 \%$ & 0,75 & $75 \%$ & 1,50 & \\
\hline 41 & $\begin{array}{l}\text { Captación, depuración y distribución de } \\
\text { agua }\end{array}$ & 0,23 & $23 \%$ & 0,45 & $45 \%$ & 0,05 & $5 \%$ & 0,88 & $88 \%$ & 1,61 & $40 \%$ \\
\hline 50 & $\begin{array}{l}\text { Comercio de vehículos automotores, } \\
\text { combustibles y lubricantes }\end{array}$ & 0,45 & $45 \%$ & 0,42 & $42 \%$ & 0,23 & $23 \%$ & 0,88 & $88 \%$ & 1,98 & $50 \%$ \\
\hline 51 & $\begin{array}{l}\text { Comercio al por mayor, excepto el comercio } \\
\text { de vehículos automotores }\end{array}$ & 0,44 & $44 \%$ & 0,42 & $42 \%$ & 0,03 & $3 \%$ & 0,66 & $66 \%$ & 1,55 & $39 \%$ \\
\hline 52 & $\begin{array}{l}\text { Comercio al por menor, excepto el comercio } \\
\text { de vehículos automotores }\end{array}$ & 0,47 & $47 \%$ & 0,42 & $42 \%$ & 0,15 & $15 \%$ & 0,87 & $87 \%$ & 1,91 & $48 \%$ \\
\hline 62 & Transporte por vía aérea & 0,88 & $88 \%$ & 0,47 & $47 \%$ & 0,17 & $17 \%$ & 0,6 & $60 \%$ & 2,12 & $53 \%$ \\
\hline 72 & Informática & 0,5 & $50 \%$ & 0,37 & $37 \%$ & 0,15 & $15 \%$ & 0,45 & $45 \%$ & 1,47 & $37 \%$ \\
\hline 73 & Centros de investigación y desarrollo & 0,7 & $70 \%$ & 0,41 & $41 \%$ & 0,34 & $34 \%$ & 0,46 & $46 \%$ & 1,91 & $48 \%$ \\
\hline 90 & $\begin{array}{l}\text { Eliminación de desperdicios y aguas } \\
\text { residuales, saneamiento y actividades } \\
\text { similares }\end{array}$ & 0,7 & $70 \%$ & 0,44 & $44 \%$ & 0,28 & $28 \%$ & 1 & $100 \%$ & 2,42 & $61 \%$ \\
\hline 851 & $\begin{array}{l}\text { Actividades relacionadas con la salud } \\
\text { humana }\end{array}$ & 0,3 & $30 \%$ & 0,43 & $43 \%$ & 0,35 & $35 \%$ & 0,56 & $56 \%$ & 1,64 & $41 \%$ \\
\hline 921 & $\begin{array}{l}\text { Actividades de cinematografía, radio y } \\
\text { televisión y otras actividades de } \\
\text { entretenimiento }\end{array}$ & 0,12 & $12 \%$ & 0,38 & $38 \%$ & 0,37 & $37 \%$ & 0,69 & $69 \%$ & 1,56 & $39 \%$ \\
\hline 8050 & Educación superior privada & 0,62 & $62 \%$ & 0,41 & $41 \%$ & 0,37 & $37 \%$ & 0,3 & $30 \%$ & 1,70 & $43 \%$ \\
\hline $551-552$ & Hoteles y Restaurantes & 0,5 & $50 \%$ & 0,44 & $44 \%$ & 0,16 & $16 \%$ & 0,65 & $65 \%$ & 1,75 & $44 \%$ \\
\hline 602-604 & $\begin{array}{l}\text { Transporte de pasajeros por vía terrestre y } \\
\text { de carga por carretera }\end{array}$ & 0,33 & $33 \%$ & 0,45 & $45 \%$ & 0,33 & $33 \%$ & 0,34 & $34 \%$ & 1,45 & $36 \%$ \\
\hline 641-642 & Correos y Telecomunicaciones & 0,13 & $13 \%$ & 0,46 & $46 \%$ & 0,06 & $6 \%$ & 0,22 & $22 \%$ & 0,87 & $22 \%$ \\
\hline $\begin{array}{l}6511- \\
6512\end{array}$ & Actividades bancarias & 0,2 & $20 \%$ & 0,36 & $36 \%$ & 0,22 & $22 \%$ & 0,11 & $11 \%$ & 0,89 & $22 \%$ \\
\hline & INDICADOR DEL SECTOR & 0,42 & $42 \%$ & 0,42 & $42 \%$ & 0,22 & $22 \%$ & 0,59 & $59 \%$ & 1,65 & $41 \%$ \\
\hline
\end{tabular}

\footnotetext{
5 Fuente: Elaboración propia.
} 
Observando el promedio obtenido en cada una de las dimensiones de la capacidad de absorción, el sector servicios presenta una capacidad de transformación del conocimiento inferior a las otras tres dimensiones, reflejando que en este sector existe una dificultad para la generación de nuevas rutinas o fuentes innovadoras para incorporar plenamente el conocimiento externo nuevo y unificarlo con el conocimiento que la empresa ya posee, lo que conlleva a pensar en la imposibilidad de generar ventajas competitivas a partir de la combinación de conocimiento nuevo y existente (González \& Hurtado, 2012).

Como dato particular, la dimensión de explotación presenta una capacidad utilizada del $59 \%$ para el sector servicios, indicando que a pesar de que se presentan innovaciones en productos y procesos, y registros de propiedad intelectual, estos aspectos no se encuentran ligados necesariamente al aprovechamiento del nuevo conocimiento adquirido.

Los resultados a nivel de subsectores, muestran tres subsectores que alcanzan un nivel de capacidad de absorción superior al 50\%: el subsector de comercio de vehículos automotores, combustibles y lubricantes $(50 \%)$; el subsector de transporte por vía aérea (53\%); y el subsector de eliminación de desperdicios y aguas residuales (61\%). El primero de estos tres subsectores hace parte del sector de autopartes y vehículos definido por el gobierno nacional como un sector de clase mundial, por ser sectores de alta demanda en la economía global, sobre los que Colombia tiene la oportunidad de tener un crecimiento superior en el corto plazo (Proexport, 2012).

El impulso de estos sectores busca mejorar la competitividad del país, aprovechando la existencia de una red de proveedores como Renault, Mazda, y General Motors, que cuentan con los más altos estándares de calidad internacional, y la presencia de un capital humano calificado dentro de la industria automotriz. Colombia se destaca como escenario ideal para generar una plataforma de fabricación y ensamble de vehículos, camiones, buses y autopartes, destinados a abastecer el mercado nacional y regional. La industria automotriz en Colombia comprende la actividad de ensamblaje (vehículos ligeros, camiones, buses y motocicletas) y fabricación de partes y piezas utilizadas en dicho proceso así como el mercado de reposición (Proexport, 2012).

En cuanto al subsector de transporte por vía aérea, su indicador de 2,12 equivale a una capacidad de absorción del $53 \%$, reflejando el acelerado crecimiento que ha tenido este subsector como consecuencia de la política de liberalización del espacio aéreo iniciada desde los años 90 , y que ha permitido la entrada de aerolíneas extranjeras a un mercado donde anteriormente solo participaban aerolíneas nacionales (Olivera et al., 2011), originando una fuerte rivalidad en precios que ha conducido a que las empresas del sector busquen nuevas formas de alcanzar ventajas competitivas, entre ellas, la búsqueda de conocimiento sobre el cliente y la generación de alianzas con el propósito de ofrecer mejores servicios a bajos costos, $y$ atraer al cliente ofreciendo beneficios y servicios adicionales.

Por último, el subsector de eliminación de desperdicios y aguas residuales demuestra el importante papel asumido tras la implementación de la política ambiental para la gestión integral de residuos peligrosos, y en particular, la implantación de los objetivos a largo plazo sobre el tratamiento de estos residuos. Dentro de las estrategias del gobierno, está el impulso a la gestión ambiental, mediante procesos de capacitación y concientización, por medio del establecimiento de centros y redes nacionales y regionales que difunden la información asociada con el manejo de residuos en empresas, con el fin de impulsar el establecimiento de programas de capacitación, control y seguimiento de la gestión ambiental (Ministerio de Ambiente, Vivienda y Desarrollo Territorial, 2005), que demuestran por qué el subsector de eliminación de desperdicios y aguas residuales posee un nivel de capacidad de absorción del conocimiento aceptable.

Dentro de los sectores con menor capacidad de absorción aparece el subsector de actividades bancarias $(22 \%)$. En Colombia, esta actividad es regulada de acuerdo a la Constitución Política de 1991, dada la necesidad de reducir cualquier tipo de riesgo relacionado con los impactos sociales y económicos de dicha actividad. Chaverra (2012) ha señalado que en los últimos años, la Superintendencia Financiera ha tenido que imponer diversas multas a las entida- 
des financieras porque no han implementado ningún procedimiento que proteja los recursos y la información de los usuarios, situación que se origina ante la carencia de prácticas organizacionales para gestionar responsablemente sus activos, coincidiendo con Martínez (2013), en la necesidad de la existencia de un modelo de gestión del conocimiento adecuado para administrar las operaciones bancarias.

Otro de los subsectores con menor capacidad de absorción es el de correos y telecomunicaciones. Estos resultados coinciden con Marulanda et al. (2013) quienes realizan un estudio sobre las tecnologías de la información y la comunicación en el eje cafetero, y encuentran que aún existen atrasos en la apropiación y uso de prácticas para la gestión del conocimiento, especialmente en todo lo relacionado con la transferencia de conocimiento y prácticas organizacionales.

En general, existe evidencia que el sector servicios alcanza a aprovechar cerca de un $50 \%$ de los conocimientos externos adquiridos para afrontar procesos de innovación y cambio organizacional (Zahra \& George, 2002), identificando que el sector posee buenas habilidades para transformar el conocimiento, pero los principales retos aparece la necesidad de transformar el conocimiento que implica la elaboración de rutinas organizacionales que permitan dar utilidad al conocimiento adquirido.

Estos resultados también deducen que sectores representativos como el subsector comercio jalonador de la economía colombiana, tampoco presenta procesos de gestión del conocimiento definidos, llevando a la necesidad de dar mayor atención al subsector de centros de investigación y desarrollo que debe estar más vinculado a promover estos procesos de adquisición y aplicación de nuevo conocimiento para propiciar la transferencia de conocimiento entre empresas e instituciones.

\section{Conclusiones e implicaciones}

En esta investigación proponemos el ICAC-Col, siendo aplicado a las empresas del sector servicios en Colombia, demostrando la existencia de un nivel insuficiente de capacidad de absorción para dicho sector. Esto permite concluir que este sector en pro- medio, cuenta con una capacidad para adquirir, asimilar, transformar y explotar el 50\% del conocimiento externo. Este porcentaje implica la carencia de habilidades y rutinas para capturar el conocimiento externo, y a pesar de que Colombia se ha caracterizado en los últimos años por mejorar su stock de conocimiento (Lugones et al., 2007), según los resultados, estos esfuerzos no han sido suficientes para incentivar apropiadamente los procesos de innovación. Esto implica, la necesidad de la formulación de políticas públicas que promuevan la transferencia de conocimiento tanto a nivel intrasectorial como intersectorial, buscando promover este proceso en los sectores reconocidos en Colombia por su mayor dinamismo, caso del sector comercio, que aunque es un sector que lidera el crecimiento económico del país, no genera procesos de avance y búsqueda de mecanismos para adquirir ventajas competitivas $y$ lograr mayor posicionamiento. Adicionalmente, en los resultados existe evidencia que los sectores que presentan una mayor capacidad de absorción, generalmente se relacionan con cambios que han debido asumir por los mismos requerimientos del mercado, o por políticas públicas que impulsaron dichos sectores, lo que demuestra la importancia del apoyo del sector público en el desarrollo de las empresas.

A partir del análisis de la capacidad de absorción con base en las cuatro dimensiones mencionadas por Zahra \& George (2002), es posible concluir que en Colombia, específicamente en los sectores analizados en esta investigación, no se puede hablar de la existencia de la capacidad de absorción del conocimiento como capacidad dinámica porque aunque se presentan en cierta medida las capacidades organizacionales de adquisición, asimilación, trasformación y explotación del conocimiento externo, estas dimensiones no trabajan de forma conjunta para obtener resultados innovadores, y adicionalmente, no es considerada su funcionalidad para el desarrollo de habilidades que permitan a las empresas crear nuevas capacidades que conduzcan a obtener ventajas competitivas. Las actividades encaminadas hacia obtener conocimiento como la inversión en $\mathrm{I}+\mathrm{D}$, la inversión en tecnología, la conformación de lazos de cooperación, no son suficientes para aprovechar el conocimiento que el entorno ofrece a las empresas, sino hay un desarrollo interno de capacidades rela- 
cionadas con la gestión humana que le den utilidad a todo el conocimiento y la información obtenida. $\mathrm{Si}$ la adquisición de conocimiento no genera ventajas competitivas, no puede concluirse que en el sector servicios en Colombia, existe una "capacidad dinámica" de absorción del conocimiento.

Teniendo en cuenta los resultados del ICAC-Col, es factible formular y reformular algunas políticas públicas orientadas al fomento de la innovación en Colombia, basadas no solo en el desarrollo de tecnologías de información, sino también en estrategias asociadas con la gestión del conocimiento para apoyar sectores importantes del país como el suministro de electricidad y gas, el sector comercial, los hoteles $y$ restaurantes, las actividades bancarias; subsectores que a pesar de ser representativos, no aprovechan adecuadamente el conocimiento externo; otros como el subsector de centros de investigación y desarrollo que son la base para impulsar los procesos de gestión del conocimiento e innovación de un país que conduzcan al crecimiento económico.

Esta investigación presenta limitaciones asociadas al alcance del estudio enmarcado en el sector servicios, dado que dentro de la muestra se excluyen algunos subsectores debido a las restricciones de selección muestral planteadas en la metodología, sin embargo la muestra sigue siendo representativa para ambos sectores. Esta es una de las limitantes por trabajar con fuentes secundarias, además de tener que construir un indicador que adapte las variables ya estimadas con el enfoque teórico utilizado. Por esta razón, son planteadas las definiciones de cada dimensión de la capacidad de absorción con base en diferentes autores, buscando hacer que las variables de la base de datos construida por el DANE, tuvieran correspondencia con los conceptos a medir. Surge entonces como línea futura de investigación, la posibilidad de construir este indicador partiendo de la elaboración de la base de datos a partir de encuestas realizadas directamente por el investigador.

Cabe aclarar que el indicador obtenido permite tener una estimación del nivel de capacidad de absorción a nivel sector, pero esto no implica que a nivel individual, algunas empresas puedan tener una capacidad de absorción inferior o superior a la calculada, siendo inadecuado generalizar estos resultados para el total de las empresas del sector. A partir de esta limitación, surge como línea futura de investigación, próximos estudios que permitan calcular la capacidad de absorción para las empresas en particular, y realizar comparaciones con los resultados obtenidos a nivel sectorial. En cuanto al instrumento de medición construido, está adaptado a la información de la Encuesta de Desarrollo e Innovación Tecnológica desarrollada en Colombia, y teniendo en cuenta que esta encuesta es basada en el Manual de Frascati y en el Manual de Oslo, es posible que sea útil con la información de otras encuestas realizadas por otros países desde estos manuales. Como líneas futuras de investigación, surge la posibilidad de utilizar este indicador para medir la capacidad de absorción en los otros sectores económicos colombianos, y establecer comparativos entre ellos. Este estudio utiliza datos de corte transversal, sería interesante observar la evolución del ICAC-Col, a lo largo del tiempo.

\section{Referencias}

Ahuja, G. \& Katila, R. (2001). Technological acquisitions and the innovation performance of acquiring firms: A longitudinal study. En: Strategic Management Journal, 22: 197-220.

Álvarez, M. (2003). Competencias centrales y ventaja competitiva: el concepto, su evolución y su aplicabilidad. En: Revista Contaduría y Administración, UNAM, 209.

Amit, R. \& Schoemaker, P. (1993). Strategic assets and organizational rent. En: Strategic Management Journal, 14(1): 33-46.

Barney, J. (1991). Firm resources and sustained competitive advantage. En: Journal of Management, 17(1): 99-120.

Calantone, R., Cavusgil, S. \& Zhao, Y. (2002). Learning orientation, firm innovation capability, and firm performance. En: Industrial Marketing Management, 31(6): 515-524.

Chaverra, M. (2012). Los 'castigados' del sistema financiero. Disponible en: http://www.dinero.com/inversionistas/articulo/ los-castigados-del-sistema-financiero/155161.

Cohen, W. \& Levinthal, D. (1990). Absorptive capacity: A new perspective on learning and innovation, Administrative Science Quarterly, 35(1): 128-152.

Cohen, W. \& Levinthal, D. (1989). Innovation and learning: the two faces of R\&D. En: Economic Journal, 99 (297): 569-596.

DANE (2011). Documento metodológico encuesta de desarrollo e innovación tecnológica en la industria manufacturera - EDIT. Recuperado de: www.dane.gov.co el 18 septiembre de 2012.

DANE (2012). Boletín de Prensa - Encuesta de desarrollo e innovación tecnológica en la industria manufacturera - EDIT V 2009-2010. Recuperado de: www.dane.gov.co el 18 de septiembre de 2012.

DANE (2013). Boletín de Prensa - Encuesta de desarrollo e innovación tecnológica en el sector servicios - EDITS III 2010-2011. Recuperado de: www.dane.gov.co el 10 de febrero de 2013.

Davila, T., Epstein, M. \& Shelton, R. (2005). Making innovation work. Upper Saddle River, NJ: Pearson Education, Inc. 


\section{PROPUESTA DE UN INDICADOR DE CAPACIDAD DE ABSORCIÓN DEL CONOCIMIENTO (ICAC-COL): EVIDENCIA EMPÍRICA PARA EL SECTOR SERVICIOS EN COLOMBIA}

De Jong, J. \& Freel, M. (2010). Absorptive capacity and the reach of collaboration in high technology small firms. En: Research Policy, 39: 47-54.

Díaz de Santos, E. (1997). Conceptos básicos. Ediciones Díaz de Santos, S. A., Madrid - España.

Dyer, J. \& Singh, H. (1998). The relational view: cooperative strategies and sources of interorganizational competitive advantage. En: Academy of Management Review, 23(4): 660 -679.

Eisenhardt, K. \& Martin, J. (2000). Dynamic capabilities: What are they? En: Strategic Management Journal, 21(10-11): 1105-1121.

Flatten, T., Engelen, A., Zahra, A. \& Brettel, M. (2011). A measure of absorptive capacity: scale of development and validation. En: EuropeanManagement Journal, 29: 98-116.

Flor, M. \& Oltra, M. (2008). La relación entre la capacidad de absorción del conocimiento externo y la estrategia empresarial: un análisis exploratorio. Trabajo presentado en el Congreso Estableciendo puentes en una economía global, Asociación Europea de Dirección y Economía, Salamanca-España.

González, C. \& Hurtado, A. (2012). La transferencia tecnológica, el capital humano y la cooperación: factores determinantes de los resultados innovadores en la industria manufacturera en Colombia 2007-2008. En: Informador Técnico, 76: 32-45.

Grant, R. (1996). Prospering in dynamically-competitive environments: organizational capability as knowledge integration". En: Organization Science, 7(4): 375-387.

Hair, J., Black, W., Babin, B., Anderson, R. \& Tatham, R. (1999). Multivariate data analysis (2th ed.). Upper Saddle River, N.J.: Pearson Education Inc.

Hooley, G., Lynch, J. \& Jobber, D. (1992). Generic marketing strategies. En: International Journal of Research in Marketing, 9(1): 75-89.

Hurley, R. \& Hult, G. (1998). Innovation, market orientation, and organizational learning: an integration and empirical examination. En: Journal of Marketing, 62: 42-54.

Jansen, J., Van Den Bisch, F. \& Volberda, H. (2005). Managing potential and realized absorptive capacity: How do organizational antecedents matter? En: Academy of Management Journal, 48(6): 999-1015.

Kane, A. (2010). Unlocking knowledge transfer potential: knowledge demostrability and superordinate social identity. En: Organization Science, 21(3): 643-660.

Lane, P. \& Lubatkin, M. (1998). Relative absorptive capacity and interorganizational learning. En: Strategic Management Journal, 19(5): 111-125.

Lane, P., Koka, B. \& Pathak, S. (2006). The reification of absorptive capacity: a critical review and rejuvenation of the construct. En: Academy of Management Review, 31(4): 833-863.

Liao, S., Wu, Ch., Hu, D. \& Tsuei, G. (2010). Knowledge acquisition, absorptive capacity, and innovation capability: an empirical study of Taiwan's knowledge-intensive industries. En: International Journal of Human and Social Sciences, 5(12): 759-766.

Lugones, G., Gutti, P. \& Le Clech, N. (2007). Indicadores de capacidades tecnológicas en América Latina. En: Serie Estudios y Perspectivas, 89, CEPAL, México.

Martínez, J. (2013). Evaluación de requerimientos para la adecuada gestión de conocimiento, caso de estudio en una unidad organizacional de soporte operativo de una entidad financiera. En: AD-minister, 23(2): 71-102.

Marulanda, C., Giraldo, J. \& López, M. (2013). Evaluación de la gestión del conocimiento en las organizaciones de la red de tecnologías de información y comunicaciones del Eje Cafetero en Colombia. En: Información tecnológica, 24(4): 105-116.

Medina, R. (2013). La ventaja competitiva como elemento fundamental de la estrategia y su relación con el sector servicios de la actividad turística. En: Observatorio de la Economía Latinoamericana, 191. Consultado el 25 de abril de 2014 en: http://www. eumed.net/cursecon/ecolat/mx/2013/ventaja-competitiva.html

Miles, R. \& Snow, C. (1978). Organizational strategy, structure and process. New York: McGraw-Hill.

Minbaeva, D., Pedersen, T., Bjorkman, I., Fey, C. \& Park, H. (2003). MNC knowledge transfer, subsidiary absorptive capacity, and HRM. En: Journal of International Business Studies, 34(6): 586-599.

Ministerio de Ambiente, Vivienda y Desarrollo Territorial (2005). Política ambiental para la gestión integral de residuos o desechos peligrosos. Dirección de desarrollo sectorial sostenible. Panamericana formas e impresos, Colombia.

Mowery, D. \& Oxley, J. (1995). Inward technologies transfer and competitiveness: the role of national innovation systems. En: Cambridge Journal of Economics, 19(1): 67-93.

Murovec, N. \& Prodan, I. (2009). Absorptive capacity, its determinants, and influence on innovation output: cross-cultural validation of the structural model. En: Technovation, 29: 859-872.

Nunnally, J. (1978). Psychometric Theory. New York: John Wiley \& Sons.

Nunnally, J. \& Bernstein, I. (1994). Psychometric theory. 3rd edition. New York: McGraw-Hill

Olivera, M., Cabrera, P., Bermúdez, W. \& Hernández, A. (2011). El Impacto del transporte aéreo en la economía colombiana y las políticas públicas. En: Cuadernos de Fedesarrollo, 34.

Penrose, E. (1959). The theory of the growth of the firm. Basil Blackwell, London.

Petroni, A. (1998). The analysis of dynamic capabilities in a competence-oriented organization. En: Technovation, 18(3): 179-189.

Poldahl, A. (2012). The two faces of R\&D: do firm's absorptive capacities matter. En: Journal of Industry, Competition \& Trade, 12(2): 221-237.

Porter, M. (1985). Competitive advantage. Free Press, New York.

Prahalad, C. \& Hamel, G. (1990). The core competence of the corporation. En: Harvard Business Review, May/June: 79-91

Proexport (2012). El periódico de las oportunidades. Recuperado el 5 de Junio de 2013 del sitio web: www.proexport.com.co

Rennie, K. (1997). Exploratory and confirmatory rotation strategies in exploratory factor analysis. Paper presented at the annual meeting of the Southwest Educational Research Association, Austin. Disponible en (consultado 17 oct de 2013): http:// mirror.eschina.bnu.edu.cn/Mirror1/accesseric/ericae.net/ft/ tamu/Rota.htm

Rodríguez, L. (1999). La innovación clave del éxito empresarial. Universidad de Vigo. La gestión de la diversidad: XIII Congreso.

Rumelt, R. (1984). Toward a strategy theory of the firm. En: Lamb, R. (ed). Competitive strategic management. Prentice Hall, Englewood Cliffs, N.J.

Sanchez, R., Heene, A. \& Thomas, H. (1996). Dynamics of competence-based competition. Oxford: Elsevier.

Schuschny, A. \& Soto, H. (2009). Guía metodológica diseño de indicadores compuestos de desarrollo sostenible. Documento de Trabajo, Comisión Económica para América Latina y el Caribe - CEPAL.

Spector, P. (1992). Summated rating scale construction: an introduction. Series: quantitative applications in the social sciences. CA: Sage Publications. 
Sullivan, D. (1994). Measuring the degree of internationalization of a firm. En: Journal of International Business Studies, 25(2): $325-42$.

Teece, D. (2007). Explicating dynamic capabilities: the nature and microfoundations of (sustainable) enterprise performance. En: Strategic Management Journal, 28(13): 1319-1350.

Teece, D. (1990). Contributions and impediments of economic analysis to the study of strategic management. En: Fredrickson, J. (ed). Perspectives on strategic management. Harper Row Publishers, Nueva York: 38-50

Teece, D. \& Pisano, G. (1994). The dynamic capabilities of firms: an introduction. En: Industrial and Corporate Change, 3 (3): 537-556

Teece, D., Pisano, G. \& Shuen, A. (1997). Dynamic capabilities and strategic management. En: Strategic Management Journal, 18(7): 509-533.

Todorova, G. \& Durisin, G. (2007). Absorptive capacity: valuing a reconceptualization. En: Academy of Management Review, 32(3): 774-786.

Tsai, W. (2001). Knowledge transfer in intraorganizational networks: effects of networks position and absorptive capacity on business unit innovation and performance. En: Academy of Management Journal, 44(5): 996-1004

Ubeda, R. \& Moslares, C. (2008). Innovando la innovación. En: Boletín económico de ICE, Información Comercial Española, No 2942.
Van Den Bosch, F., Volberda, H. \& De Boer, M. (1999). Co-evolution of firm absorptive capacity and knowledge environment: organizational forms and combinative capabilities. En: Organization Science, 10(5): 551-568.

Ventura, V. (1996). Análisis dinámico de la estrategia empresarial: un ensayo interdisciplinar. Servicio de publicaciones. Universidad de Oviedo.

Wang, C. \& Ahmed, P. (2007). Dynamic capabilities: a review and research agenda. En: International Journal of Management Reviews, 9(1): 31-51.

Wernerfelt, B. (1984). A resourced based view of the firm. En: Strategic Management Journal, 5(2): 171-180.

Woiceshyn, J. \& Daellenbach, U. (2005). Integrative capability and technology adoption: evidence from oil firms. En: Industrial and Corporate Change, 14(2): 307-342.

Zahra, S., Ireland, D. \& Hitt, M. (2000). International expansion by new venture firms: international diversity, mode of market entry, technological learning, and performance. En: Academy of Management Journal, 43: 925-950.

Zahra, S. \& George, G. (2002). Absorptive capacity: a review, reconceptualization, and extension. En: Academy of Management Review, 27(2): 185-203.

Zhao, H., Tong, X., Wong, P. \& Zhu, J. (2005). Types of technology sourcing and innovative capability: an exploratory study of Singapore manufacturing firms. En: Journal of High Technology Management Research, 16: 209-224.

Zollo, M. \& Winter, S. (2002). Deliberate learning and the evolution of dynamic capabilities. En: Organization Science, 13(3): 339-351. 